\title{
Introduction
}

\section{Annette Kur*}

Is there a coherent system underlying the entire field of intellectual property (IP), or are the different constituent areas only loosely connected with each other? Have the foundations on which the different rights were originally grounded converged, or are they drifting apart? Does the fact that the law must react to a host of problems characterised by an ever-increasing degree of sophistication necessarily entail fragmentation ultimately leading to inconsistency, or are the principles informing legal choices sustainable enough to provide a solid and resilient frame? Vice versa - are those principles too rigid and inflexible, and therefore incapable of responding to novel challenges? To what extent, and where, should the system make room for more differentiation? Which tools does the law provide for the adjustment of protection to different needs and circumstances, and how much flexibility exists in employing those tools? Can One Size Fit All?

The idea of focusing on these and other issues at ATRIP meetings was born out of the Intellectual Property of Transition project, to which Graeme Dinwoodie refers in the first chapter of this book. The clue was taken from two seemingly antagonistic tendencies that were considered characteristic of contemporary IP law. As their overall coverage broadens, legal fields such as patent and copyright law become increasingly compartmentalised: pharmaceutical patents have little in common with patents in 'classical' technical fields such as machinery, and copyright in works of fine art is quite another discipline than copyright in news articles or computer programs. In order to attain and keep up expert status on a particular subject, a high degree of specialisation is needed, which may impede a full view on IP as one coherent field. On the other hand, there is also an increasing inclination towards overlaps, both as regards the way in which one and the same object may attract cumulative protection under different laws, and regarding the legal objectives underlying the different fields.

* Senior Researcher and Head of Unit, Max Planck Institute for Intellectual Property and Competition Law, Munich; President of ATRIP 2007-09. 
While patent and copyright protection were originally based on quite distinct motives and concepts, patent law - in some parts of the world has ventured out of the field of technology, while copyright long ago left the sphere of purely artistic creations. Although traditional rhetoric may still convey more idealistic views, legal developments in all fields are predominantly driven by utilitarian motives. Protection and encouragement of financial investment have become the primary rationale for the creation of new and extension of existing rights. Protection is frequently tailored so as to prevent third parties from taking commercial advantage of achievements made by the first investor, irrespective of whether or not a risk of market failure is created thereby. The problem with that approach is that it lacks an inherent balancing element, and that it tends to respond in an ad hoc fashion to needs and demands which are voiced strongly enough to create political pressure.

One possible response to those trends would be to embark on a quest for a 'One Right' system, which could provide the groundwork for establishing a well-calibrated IP regime. The task would be to identify the features of a core right which extends from a nucleus of unconditional power to exclude others from accessing and using the protected subject matter over various forms and degrees of attenuation to full accessibility and free use for everyone. The architecture of such a system would have to be elaborated on the basis of a holistic approach, investigating the commonalities in the foundations as well as in the existing and/or desirable limitations of different types of rights. For instance, an attempt could be made to draw up a charter of 'users' rights' forming the baseline for third-party conduct which must regularly be deemed permissible, irrespective of the kind of right invoked.

Of course, the outcome of that exercise would be deplorable if it left less room than at present for the finetuning of protection. However, depending on the details of implementation, the system might even allow for an optimum of flexibility and differentiation, with the appraisal being based on a common catalogue of evaluation factors interacting with each other. In its ideal form, the system could be compared to a tree, where one strong and massive trunk grows out of a meshwork of roots, then separates into different branches which sprout a large number of twigs, and finally end up in smaller and smaller sprigs which are too numerous to be counted. Translated into law, this means that full room is given for consideration of the specificities found in individual cases, while nevertheless, all solutions must respond, and must conform to, the common objectives and principles that provide the backbone of the system, so as to avoid arbitrary and inconsistent decisions.

While flexibility would thus be preserved or even enhanced, an obvious 
drawback of such a system would be that it tends to reduce legal certainty and foreseeability, and that it imposes a very high burden on those who are responsible for its application. It would also require the revisiting and, where necessary, the adaption of the legislative techniques traditionally employed in intellectual property law in order to give more room to individual evaluations.

Apart from such ambitious goals, a modest and probably more realistic aim for a holistic approach towards IP would be to promulgate an enhanced form of an Intellectual Property Code, which contains common rules on (administrative) procedures and sanctions as well as, for example, on IP rights as objects of property, where that is feasible. This might also help to sharpen the perception of the common features of legal fields which have remained separate up till now, and of the need for more differentiated treatment in others.

The contributions in this book address various aspects on the spectrum of issues arising from one or another form of a 'One Right' approach. They look into the underlying policies and economic foundations of intellectual property right, the principles governing limitations and exceptions, ownership, transfer and other contractual matters. Finally, they also address the question of whether one size can fit all with regard to its implications for intellectual property law. The conclusions drawn and suggestions made may differ in their potential to question current legal concepts and solutions. However, they are all proof of the fact that to conceive of intellectual property as one common area lending itself to horizontal thinking is a valuable exercise in legal analysis, and may foster new insights. 
Annette Kur and Vytautas Mizaras - 9780857931542 Downloaded from PubFactory at 04/26/2023 01: 08:26PM via free access 